\title{
Scattering Theory of Kondo Mirages and Observation of Single Kondo Atom Phase Shift
}

\author{
Gregory A. Fiete ${ }^{1}$, Jesse S. Hersch ${ }^{1}$ and Eric J. Heller ${ }^{1,2}$ \\ ${ }^{1}$ Department of Physics, Harvard University, Cambridge, Massachusetts 02138 \\ ${ }^{2}$ Department of Chemistry and Chemical Biology, Harvard University, Cambridge, Massachusetts \\ 02138 \\ H.C. Manoharan, C.P. Lutz, and D. M. Eigler \\ IBM Research Division, Almaden Research Center, 650 Harry Road, San Jose, California 95120
}

\begin{abstract}
We explain the origin of the Kondo mirage seen in recent quantum corral Scanning Tunneling Microscope (STM) experiments with a scattering theory of electrons on the surfaces of metals. Our theory combined with experimental data provides the first direct observation of a single Kondo atom phase shift. The Kondo mirage observed at the empty focus of an elliptical quantum corral is shown to arise from multiple electron bounces off the corral wall adatoms in a manner analagous to the formation of a real image in optics. We demonstrate our theory with direct quantitive comparision to experimental data.
\end{abstract}

PACS numbers: 73.20.At, 72.10.Fk, 72.15.Qm, 3.65.Nk

The Kondo effect is a fascinating, many-body quantum phenomenon occuring at low temperatures, whereby a magnetic impurity in metallic bulk or on a metallic surface has its local moment screened by a cloud of conduction electrons, called the Kondo cloud, that 
forms in its vicinity [四]. Until recently, only bulk measurements were possible on Kondo systems, leaving isolated Kondo atoms and their scattering phase shifts unstudied. The atomic resolution of the STM now makes the study of single, isolated Kondo atoms possible [2]. Kondo adatoms are identified by a sharp ( $\sim 10 \mathrm{meV}$ wide $)$ feature in the differential tunneling conductance $(d I / d V)$ as a function of STM tip bias with respect to the surface of the material. The feature typically appears as a dip near the Fermi energy $\left(E_{F}\right)$ in $d I / d V$, is localized to within $\sim 10 \AA$ of a Kondo impurity and is only observed at low temperatures $(\sim 4 \mathrm{~K})$. If the temperature of the substrate becomes to high $(\sim 100 \mathrm{~K})$, the correlations between the impurity spin and the conductions electrons are broken and the Kondo signature disappears.

Landmark STM experiments have recently discovered the remarkable fact that when a Kondo atom is placed at one focus of a properly sized empty elliptical quantum corral built from Kondo adatoms, a "mirage" of the Kondo feature is cast to the opposite focus [2] more than $70 \AA$ away. Since the Kondo effect arises from electron correlations, what does the Kondo feature at the unoccupied focus imply about local electron correlations there? An important feature of the mirage experiments is that they were done on the surface of $\mathrm{Cu}(111)$ which is known to have surfaces states which act as a 2-dimensional electron gas. The central result of this paper is to present a theory of the Kondo mirage based on electrons in this surface state scattering to infinite order from all Kondo atoms-both the Kondo atoms that make up the walls of the corral and the Kondo atom that sits at the focus. The theory is valid for all arrangements of adatoms, whether arranged into a corral shape or any other arbitrary structure including "open" structures. Electron scattering on the surface can be directly related to the $d I / d V$ of the STM measurements. Thus, by solving the electron scattering problem on the surface in the presense of impurities we can compute the STM conductance [5].

The basic Hamiltonian in the Kondo problem is given by

$$
H=\sum_{\mathbf{k} \sigma} \epsilon_{k} c_{\mathbf{k} \sigma}^{\dagger} c_{\mathbf{k} \sigma}+\frac{J}{N} \sum_{\mathbf{k}, \mathbf{k}^{\prime} \sigma \sigma^{\prime}} \mathbf{S} \bullet \mathbf{s}_{\sigma \sigma^{\prime}} c_{\mathbf{k} \sigma}^{\dagger} c_{\mathbf{k}^{\prime} \sigma^{\prime}}
$$


where $\epsilon_{k}$ is the dispersion relation (given by band structure calculations or experiment) of the conduction electrons, $c_{\mathbf{k} \sigma}^{\dagger}$ is an operator that creates an electron in the state $\mathbf{k}$ with spin $\sigma$ and $c_{\mathbf{k} \sigma}$ destroys an electron in the state $\mathbf{k}$ with spin $\sigma$. This first term describes the energy of free conduction electrons. The second term describes spin-flip scattering processes where a conduction electron flips its spin and the impurity changes its spin in response during a scattering event. $N$ is the number of sites in the lattice, $J$ is the coupling constant, $\mathbf{S}$ represents the impurity spin and $\mathbf{s}_{\sigma \sigma^{\prime}}$ represents the conduction electron spin. It is well known that the Kondo effect occurs when $J>0$ [困] and the low energy properties of this Hamiltonian are described by an effective Hamiltonian with $J \rightarrow \infty$ so that the conduction electrons become "locked" into an anti-aligned state with the impurity moment. Spin-flip process are thus frozen out. We do not attempt to solve this Hamiltonian, but assume that we are working in the low temperature regime where spin flips are frozen out as experiment suggests from the feature in $d I / d V$ described earlier.

In the Fermi liquid description of Kondo impurities below their Kondo temperature, $T_{K}$, the impurities may be characterized by a scattering phase shift that they impart to impinging electrons (quasi-particles) [6]. For $T<<T_{K}$ Kondo impurities act as potential scatterers with all the many-body physics appearing as an energy scale in the (resonant) scattering phase shift [1]:6]. We apply our theory to experimental data to extract the phase shift with full energy dependence of a single Kondo atom and report its value for the first time ever and present calculations that show beautiful quantitative agreement with experiment. Our theory clarifies what the STM does and does not measure in mirage experiments regarding local correlations of electrons. In particular, our theory suggests that the mirage experiments do not probe local electron-electron correlations because the Kondo correlations only appear in the theory as an energy scale, $T_{K}$, in the scattering phase shift (or alternatively, in the density of states).

We emphasize that our theory is phenomenological in that we do not attempt to compute the tunneling conductance from first principles on top of an atom as was done in [0,8]. Our theory is based on electron scattering which is by its nature asymptotic-an electron comes 
in, strikes a Kondo adatom, and leaves with a phase shift. Therefore, our theory only makes accurate quantitative predictions when the STM tip is more than $7 \AA$ away laterally from a Kondo adatom on the surface. This is precisely the region where we can make accurate predictions about the quantum mirage with our theory. When the STM tip is within $7 \AA$ laterally of an impurity there is accumulated screening charge and orbital density present that is not accounted for in our theory and thus the theory has no predictive power there.

When an STM tip is biased negatively with respect to the surface of a metal, such as $\mathrm{Cu}(111)$, electrons can tunnel from the tip onto the surface, creating a region of enhanced electron amplitude under the tip which travels outwards as a wave on the surface. This electron wave may encounter surface defects such as adatoms or step edges, which cause scattering. Part of the scattered wave returns to the STM tip where, depending on the relative phase of the outgoing and incoming amplitudes, it interferes constructively or destructively with the outgoing wave. This interference leads to fluctuations in the STM current as the tip is moved over the surface. The STM, then, is sensitive to the coherent amplitude that is reflected from defects on the surface. Heller et al. [5] showed that because the Fermi wavelength of the surface state electrons on $\mathrm{Cu}(111)$ is much larger than the size of adatoms and because the adatoms are separated by a distance large compared to their size, it is permissible to use a multiple s-wave scattering expansion to calculate the electron amplitude on the surface. In this picture, the scattered electron wave, and therefore the STM signal, are determined by a single quantity: the s-wave phase shift of the scattered wave, $\delta_{o}(\epsilon)$, which will typically by complex. The imaginary part of the phase shift represents "absorption" (incoherent scattering of electrons) by the adatoms which tend to couple surface states to bulk states [5.9:10] resulting in a loss of electrons from the surface states. The loss of electrons from the surface states at impurities has measurable consequences in STM experiments and is related to the attenuation of the mirage at the empty focus of an ellipse relative to the Kondo feature at the opposite, occupied focus.

To compute $d I / d V$ from a scattering calculation, we follow the method of Heller et al [5]. For s-wave scattering from a single adatom in two dimensions, the wave function is 
[11], $\psi(\mathbf{r}) \rightarrow \phi(\mathbf{r})+f \frac{e^{i\left(k r-\frac{\pi}{4}\right)}}{\sqrt{r}}$, where the scattering amplitude, $f$, is $f=\frac{e^{2 i \delta_{o}(\epsilon)}-1}{\sqrt{2 \pi k}} . \phi(\mathbf{r})$ is the amplitude of the circular electron wave emanating from the STM tip. There is no angular dependence of $f$ in the s-wave approximation. $k=\frac{2 \pi}{\lambda}$, where $\lambda$, the wavelength of the electrons on $\mathrm{Cu}(111)$, is $29.5 \AA . \epsilon(k)=\frac{\hbar^{2} k^{2}}{2 m^{*}}$ is known from the surface state electron dispersion relation of $\mathrm{Cu}(111)$ which has an effective mass of $m^{*}=0.38 m_{e}$. Once $\delta_{o}(\epsilon)$ is determined, the surface scattering can be computed. In the limit of small bias voltages and low temperature [12],

$$
\frac{d I}{d V} \propto \operatorname{LDOS}(\vec{r}, \epsilon)=\sum_{\nu}\left|\psi_{\nu}(\vec{r})\right|^{2} \delta\left(E_{\nu}-\epsilon\right),
$$

where $\epsilon$ is the energy determined by the bias voltage $\mathrm{V}$ and the Fermi energy $\mathrm{E}_{F}$, via $\epsilon=\mathrm{E}_{F}+e \mathrm{~V} ; \nu$ labels the scattering eigenstates in the presence of the adatom. When several or many adatoms are present as in this paper, a multiple scattering approach [5] is used to compute $\operatorname{LDOS}(\vec{r}, \epsilon)$. By definition the LDOS is related to the Green's function by $\operatorname{LDOS}(\vec{r}, \epsilon)=-\frac{1}{\pi} \operatorname{Im}\left[G_{r e t}(\vec{r}, \epsilon)\right]$, where

$$
G_{r e t}=G_{o}+G_{o} T G_{o}
$$

is the retarded Green's function and $T$ is the T-matrix whose dimensions are $\mathrm{N}$ by $\mathrm{N}$ when there are $\mathrm{N}$ scatterers present. The T-matrix contains all the information about the physical positions of the scatters relative to each other and their corresponding phase shifts which could be different for each scatterer. $G_{o}(\vec{r}, \epsilon)$, the free electron's Green's function in two dimensions. Thus, $d I / d V$ of the STM may be obtained by solving the scattering problem.

Our theory, then, involves the following approximations, assumptions and limitations: (i) We can characterized the "target" (adatom) by a single parameter, the s-wave phase shift and this must be determined from experiment or otherwise (ii) The internal degrees of freedom (spin) of the Kondo adatoms are frozen out at the temperature of the experiment $(\sim 4 \mathrm{~K})$ so we may use the results of Nozieres [6] to treat the Kondo atom as a potential scatterer with a phase shift (iii) The adatoms are far enough apart so that we may treat the electron propagation between them as free and that RKKY interactions are sufficiently weak 
so that the single-impurity Kondo physics is not altered (iv) The theory does not include any non-equilibrium effects and does not treat the charge density right at an atom correctly.

To make a direct comparison with experiment, we must obtain the phase shift of the Kondo adatoms. We do not have an ab initio calculation of the phase shift of a single Co adatom. Rather, we fit the resonant form of the phase shift, including inelasticity, and calculated the multiple scattering problem with this single atom data. We emphasize that we have not blithely ignored the Kondo effect of the wall and focal adatoms in our phenomenological fit to the scattering phase shift. We have used the fact that the spin-flips have been frozen out to lowest order at the temperatures of the experiment and that the Kondo effect appears through the energy scale of the resonant spectral peak in the density of states near $E_{F}$ [司].

Since the on-atom electron orbital density is not accounted for in scattering theory, we used an on-atom fit involving only a renormalization of the free-space Green's function and a change in the background phase shift to compute the STM signal on top of a Kondo adatom [7.:8, 14]. This on-atom fit is not part of our theory, but only a means of setting a reference point between on-atom density not accounted for in our theory and the electron density anywhere more than $7 \AA$ away from an atom on the surface which $i$ accounted for properly in our theory. This fit in no way compromises our fundamental result that the mirage is due to resonantly scattering electrons from the Kondo atoms of the walls and focus. It is used only as a method to determine as accurately as possible the phase shift of the $\mathrm{Co}$ on $\mathrm{Cu}(111)$. Determining the phase shift this way from experimental data constitutes a measurement of the single Kondo atom phase shift for the first time.

We find a good fit to the s-wave scattering phase shift to be $\delta_{o}(\epsilon)=\delta_{b g}+i \delta^{\prime \prime}+\tan ^{-1}\left(\frac{\epsilon-\epsilon_{o}}{\Gamma / 2}\right)$, where $\delta_{b g}=\frac{\pi}{4} \pm \frac{\pi}{10}, \delta^{\prime \prime}=\frac{3}{2} \pm \frac{1}{4}, \Gamma=(9 \pm 1) \mathrm{meV}$ and $\epsilon_{o}=E_{F}-1 \mathrm{meV}$ are determined by experiment. $\delta_{b g}$ is a background phase shift (possibly due to static charge screening at the impurity) that controls the resonant line shape of the adatom scattering cross-section. $\delta^{\prime \prime}$ is the imaginary part of the phase shift and is a measure of the inelasticity in adatom 
scattering. $\operatorname{Tan}^{-1}\left(\frac{\epsilon-\epsilon_{o}}{\Gamma / 2}\right)$ reflects resonant scattering due to the presence of Kondo physics. A similar form of phase shift has been derived by Ujsaghy from a more microscopic point of view in [14]. The narrow spectral peak near $E_{F}$ leads to resonant scattering Fermi surface electrons and sets the scale of the resonance in the phase shift. It is likely that both bulk and surface states are participating in the Kondo effect at an adatom, but the STM signal is dominated by the surface state Kondo effect in the regime of validity of our theory $(>7$ $\AA$ away from adatom).

Applying the theory to elliptical corrals results in the images shown in Fig. 1 and Fig. 2. The agreement with experiment is excellent. Our calculation of the tunneling spectrum at the two foci is compared with experiment in Fig. 3. Note that the signal at the unoccupied focus is attenuated by approximately a factor of 8 , both experimentally and theoretically. $\delta^{\prime \prime}$ is largely responsible for the attenuation of the mirage at the unoccupied focus of the ellipse: the rest of the attenuation comes from flux leaking through the corral. The calculated spectroscopy in Fig. 3 most clearly demonstrates that the Kondo mirage is due to resonant scattering of electrons from the adatom at the opposite focus. (Even though the electrons are also resonantly scattering from the wall adatoms. Our calculations show that the wall atoms' Kondo resonances play no essential role in the projection of the mirage to the empty focus. Walls with $\delta=i \infty$ [5] also result in the mirage at the empty focus provided the focal adatom is treated as a resonant scatterer. Experimentally the same result is found [2].)

The Kondo mirage results from resonant scattering at the Kondo adatoms and from the geometry of the quantum corral. Although any ellipse will focus rays at the foci, but only certain sized ellipses will give a good mirage effect-those which have large surface state amplitude at the foci when the scattering problem is calculated and this depends on the relative size of the ellipse and $\lambda_{F}$. Only then will there be appreciable surface state electron amplitude at the focal adatom to give a Kondo effect in the surface states of $\mathrm{Cu}(111)$. Our theory predicts that the Kondo mirage is not restricted to an ellipse or even a "closed" structure. Any time one can construct an arrangement of adatoms or other defects that lead to a build up of surface state electron amplitude at two locations within the electron's 
coherence length, a mirage can be projected.

Returning to our original question of whether the mirage reveals any information about local correlation, we conclude that it does not. There is no explicit information about electron correlations in our theory, which gives remarkable agreement with experiment in reproducing the Kondo mirage and standing wave patterns in the elliptical corral. We are thus led to the conclusion that the mirage at the empty focus of the elliptical corral is not a result of electron correlations under the tip, but rather resonant scattering of electrons from the focal adatom and scattering (resonantly or not) from the adatoms of the walls of the corral. Intuitively this makes sense because the electrons tunneling out of the STM are unpolarized so it is not possible for the STM to give any direct information about electron correlations. The unpolarized STM only returns an average signal of spin up and spin down electrons tunneling into the surface.

In summary believe that the term "mirage" is very apt indeed: the Kondo effect appears to be projected to a remote spot, but the STM is only probing the Kondo focal adatom from afar. The remote image of the Kondo effect is explained quantitatively by the refocusing of the s-wave electrons leaving the empty focus and traveling to the occupied focus after bouncing off the walls.

Is the Kondo effect really projected to the empty focus? Yes, and no. One need look no further than the concept of the real image in optics to understand the sense in which projection occurs.

We acknowledge enlightening discussions with B.I. Halperin, S. Kehrein, and Y. Oreg. This research was supported by the National Science Foundation under Grant No. CHE9610501 and by ITAMP.

While preparing this manuscript we became aware of several papers on a similar topic: Weissman et al, cond-mat/0007485; Porras et al, cond-mat/0007445; and Agam et al condmat/0006443. 


\section{REFERENCES}

[1] A.C. Hewson, The Kondo Problem to Heavy Fermions. (Cambridge University Press, Cambridge, 1997).

[2] H.C. Manoharan, C.P. Lutz, D.M. Eigler, Nature, 403, 512 (2000).

[3] J. Li et al., Phys. Rev. Lett. 80, 2893 (1998).

[4] V. Madhavan et al., Science 280, 567 (1998).

[5] E.J. Heller et al., Nature 369, 464 (1994).

[6] P. Nozieres, J. Low Temp. Phys., 17, 31 (1974).

[7] T. Kawasaka et al., J. Appl. Phys. 86, 6970-6974 (1999).

[8] A. Schiller, S. Hershfield, Phys. Rev. B 61, 9036 (2000).

[9] G. Hormandinger, J.B. Pendry, Phys. Rev. B. 50, 18607 (1994).

[10] S. Crampin, M.H. Boon, J.I. Inglesfield, Phys. Rev. Lett. 73, 1015 (1994).

[11] L.D. Landau, E.M. Liftshitz, Quantum Mechanics (Butterworth-Heinemann, Oxford, 1997).

[12] J. Tersoff, D.R. Hamann, Phys. Rev. B 31, 805 (1985).

[13] M.F. Crommie, C.P. Lutz, D.M. Eigler, Science 262, 218 (1993).

[14] O. Ujsaghy, et al., cond-matt/0005166.

Fig. (1). Topograph standing wave patterns of a Kondo corral. Using the scattering theory and phase shifts described in the text, these STM topograph images were computed using exact $\mathrm{Co}$ adatom positions on $\mathrm{Cu}(111)$ at $4 \mathrm{~K}$. The agreement between theory (a, $\mathbf{c}$ and $\mathbf{e}$ ) and experiment ( $\mathbf{b}, \mathbf{d}$ and $\mathbf{f})$ is remarkable. All the experimental images have 
been symmetrized by adding the image to itself after being reflected about its major axis. Topographic images were calculated by numerically integrating the $\operatorname{LDOS}(\vec{r}, \epsilon)$ over $\epsilon$ for $E_{F} \leq \epsilon \leq E_{F}+10 \mathrm{mV}$. This corresponds to the topographic images taken experimentally in $\mathbf{b}$ and $\mathbf{d}$ at a bias voltage of $10 \mathrm{mV}$. $\mathbf{e}$ is the difference of $\mathbf{a}$ and $\mathbf{c}$. $\mathbf{f}$ is the difference of $\mathbf{b}$ and $\mathbf{d}$.

Fig. (2). $d I / d V$ Standing wave patterns of a Kondo corral. Same theory vs. experiment arrangment as in Fig. (1). $d I / d V$ measurements were taken simultaneously with topographic images at a $10 \mathrm{meV}$ bias. Note that $\mathbf{e}$ and $\mathbf{f}$ resemble an eigenstate of the ellipse. The ellipse was constructed to have large surface state amplitude at the two foci.

Fig. (3). Tunneling into the focal atom and empty focus: The Mirage. Tunneling spectroscopy is calculated (dashed lines) with the scattering theory and phase shift given in the text at the empty focus a. Tunneling spectroscopy at the occupied focus is shown in $\mathbf{b}$. A constant background slope has been removed from both the experimental data and the calculation. The attenuation of the mirage is determined by inelasticity in the scattering of electrons at the walls of the ellipse. The theoretical signal $5 \AA$ away from the empty focus in $\mathbf{a}$ is lost in the noise of the experiment. 
FIGURES
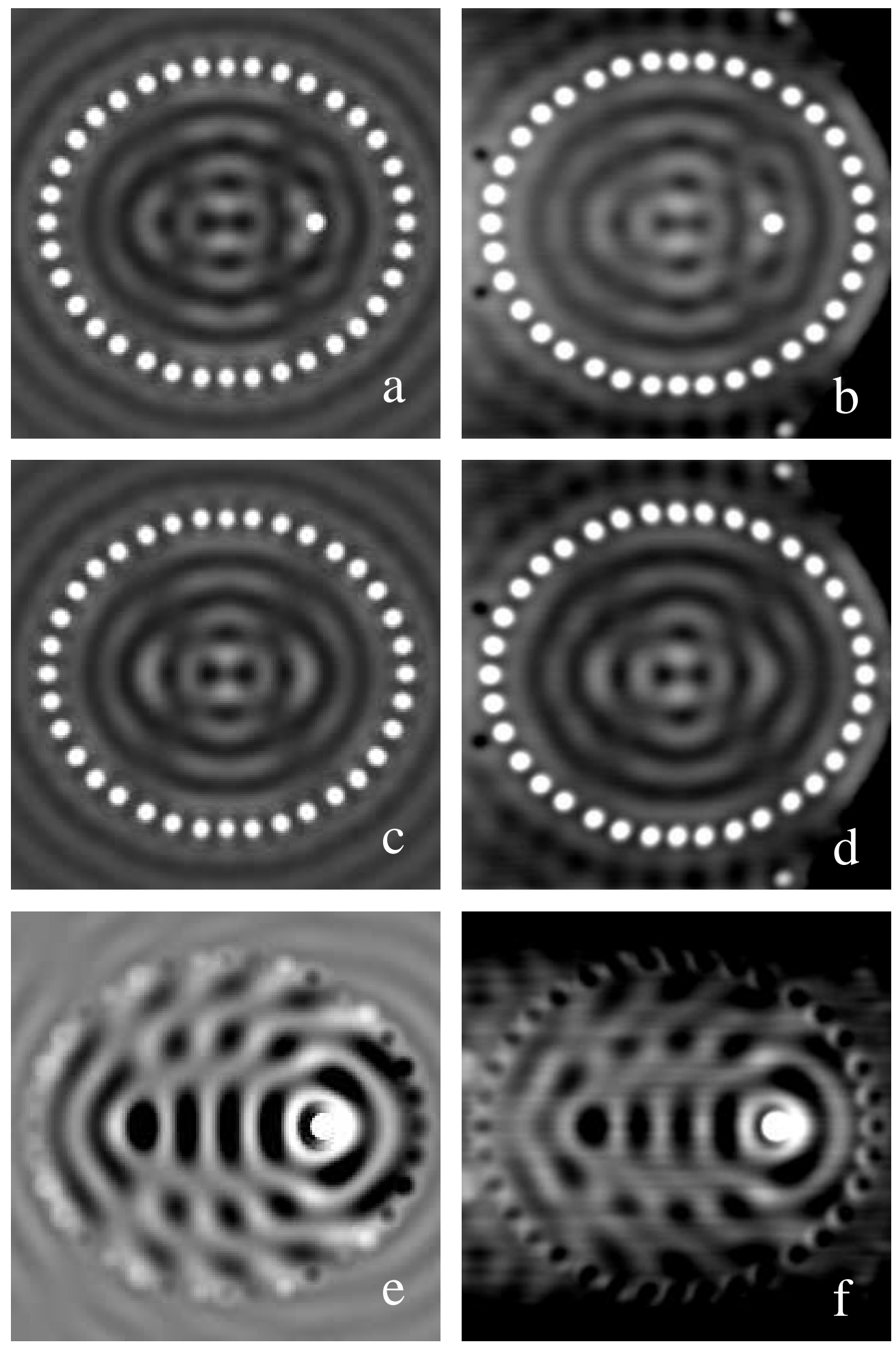

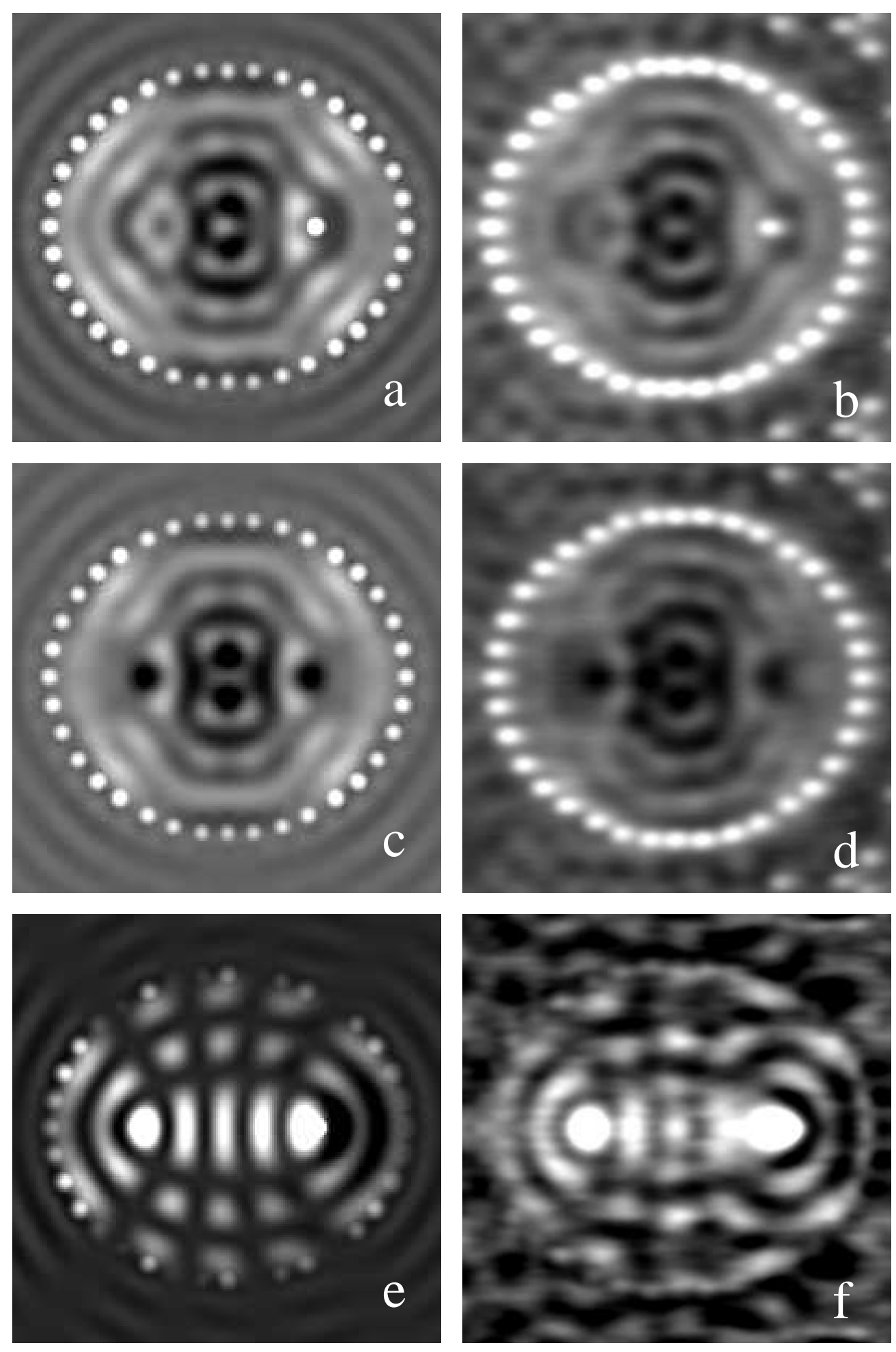


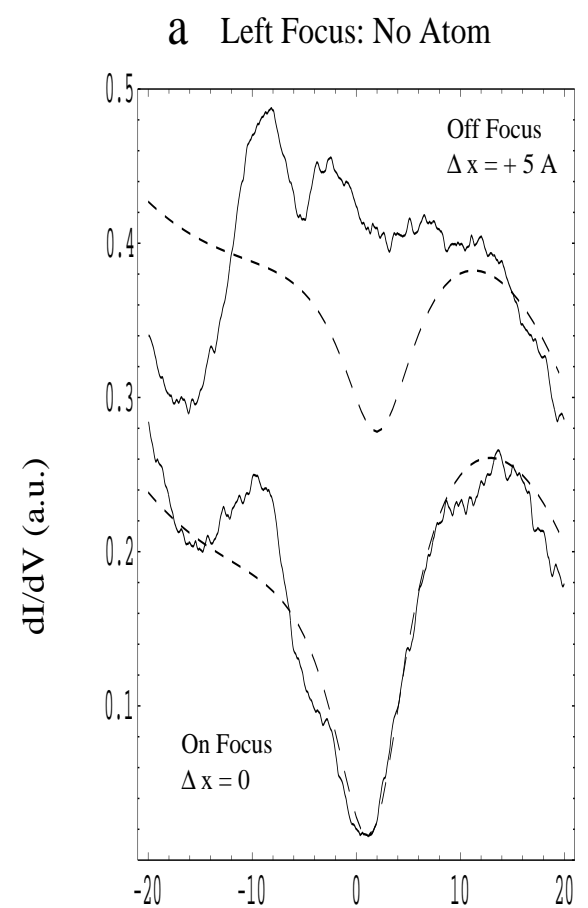

Sample Bias V (mV)

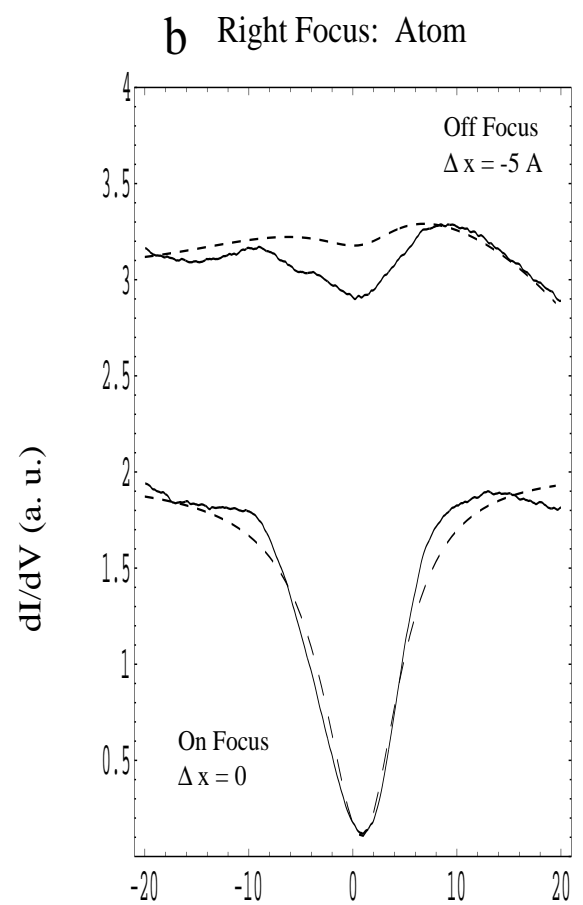

Sample Bias V (mV) 